\title{
Clinical factors that affect the pregnancy rate in frozen- thawed embryo transfer in the freeze-all policy
}

\author{
Seo Yoon Hwang, Eun Hye Jeon, Seung Chul Kim, Jong Kil Joo \\ Department of Obstetrics and Gynecology, Pusan National University Hospital, Pusan National University School of Medicine, Busan, Korea
}

Received: May 21, 2019

Revised: September 3, 2019

Accepted: September 11, 2019

Corresponding author:

Jong Kil Joo

Department of Obstetrics and

Gynecology, Pusan National

University School of Medicine, 179

Gudeok-ro, Seo-gu, Busan 49241,

Korea

Tel: $+82-51-240-7287$

Fax: +82-51-248-2384

E-mail: jongkilj@hanmail.net
Background: This study was conducted to analyze clinical factors that can affect pregnancy rates in normal responders undergoing the freeze-all policy in in vitro fertilization.

Methods: We evaluated 153 embryo transfer cycles in 89 infertile women with normal response to controlled ovarian stimulation (COS). After COS, all embryos were cultured to the blastocyst stage, and good quality blastocysts were vitrified for elective frozen-thawed embryo transfer (FET). Clinical variables associated with $\operatorname{COS}$ and the results of COS and culture, including the number of retrieved oocytes, fertilized oocytes, and frozen blastocysts were compared between the pregnant group and the non-pregnant group.

Results: After a single cycle of COS for each patient, 52 patients became pregnant while 37 did not. Significant differences were observed in the number of matured oocytes, fertilized oocytes, frozen blastocysts, and transferred embryos. The number of frozen blastocysts in the pregnant group was almost twice that in the non-pregnant group (5.6 \pm 3.1 vs. $2.8 \pm 1.9, p<0.001)$. The area under the receiver operating characteristic curve for the 4 frozen blastocysts was 0.801 in the pregnant group.

Conclusion: In the freeze-all policy, the number of matured oocytes, number of fertilized oocytes, and number of frozen blastocysts might be predictive factors for pregnancy.

Keywords: Blastocyst; Embryo transfer; In vitro fertilization; Pregnancy

\section{Introduction}

A clear trend has been observed toward increase in frozen-thawed embryo transfers (FETs) [1]. It might be due to the increase in the live birth rates with FET when compared with fresh transfers and improved perinatal outcomes in FET [2]. Suggested mechanisms of the benefits of FET are the synchrony of embryo-endometrial development and the physiological concentration of estradiol and progesterone in the FET cycle. In fresh embryo transfer cycle, the concentrations of estradiol and progesterone surpass the physiologic levels of normal menstrual cycle or the FET cycle.

The freeze-all policy was introduced based on these findings
[3]. Originally, the freeze-all policy was based on the segmentation of in vitro fertilization (IVF) treatment with the use of a gonadotropin-releasing hormone ( $\mathrm{GnRH}$ ) antagonist protocol, $\mathrm{GnRH}$ agonist triggering, the elective cryopreservation of all embryos by vitrification, and FET in the subsequent cycle [4].

Although the freeze-all policy might be a promising new method to overcome the old problems in the IVF procedure, there are some weaknesses and threats. In poor responders, the embryos may not be developed to an appropriate stage of freezing. In such cases, clinicians may not retrieve any embryos for transfer and it would affect the confidence between the patients and the clinicians. Moreover, the exact parameters related with the pregnancy

Copyright(C) 2020 Yeungnam University College of Medicine

This is an Open Access article distributed under the terms of the Creative Commons Attribution Non-Commercial License (http://creativecommons.org/licenses/by-nc/4.0/) which permits unrestricted non-commercial use, distribution, and reproduction in any medium, provided the original work is properly cited. 
rate in the FET cycle are unclear. Therefore, it is important to predict the possibility of acquisition of good quality embryos and the pregnancy rates of the FET cycle.

In a recent study, characteristics of high-quality embryos, endometrial preparation protocol, number of transferred embryos, and body mass index of the patients independently affected the live birth rate in FET [5]. However, in case of the freeze-all policy, all embryos are frozen and fresh embryo transfer is not performed. Hence, the parameters primarily related with the pregnancy rate are unclear.

In this study, we analyzed the differences in various clinical parameters in the freeze-all policy between the pregnant group and the non-pregnant group through a single cycle of controlled ovarian stimulation (COS) composed of 3 cycles of FET.

\section{Materials and methods}

\section{Ethics statement}

The present study was approved by the Institutional Review Committee of Pusan National University Hospital (IRB No. H-1908-021-082). Eighty-nine infertile women qualified as normal responders were recruited for this study and provided informed consent. The informed consent included the benefits and the threats of the freeze-all policy.

\section{Patients}

This study was retrospectively conducted from January 2015 to December 2016 at the Infertility Center, Pusan National University Hospital. A normal responder was defined as having the following criteria: (1) age < 40 years, (2) follicle stimulating hormone level $<12 \mathrm{mIU} / \mathrm{mL}$ at cycle day 2 or 3 and, (3) anti-Müllerian hormone level $>1 \mathrm{ng} / \mathrm{mL}$. Patients having inappropriate endometrium for implantation, which included conditions such as endometrial synechiae, unresponsive thin endometrium, or abnormal anatomy of the uterine cavity, were excluded. After infertility workups, endometrial and/or tubal corrective surgeries were performed after COS and before FET in indicated cases. This study was conducted through a single cycle of COS composed of 3 cycles of FET.

\section{Preparing the blastocyst and the endometrium for frozen-thawed embryo transfer}

We adopted the routine protocol of our clinics for COS, the blastocyst culture, and the preparation of the endometrium [6]. The infertile women individually received the GnRH antagonist protocol or the GnRH agonist long protocol. For triggering, human chorionic gonadotropin (hCG) (Ovidrel 5,000-10,000 IU; Mer- ck Serono, Geneva, Switzerland) or a GnRH agonist (decapeptyl $0.2 \mathrm{mg}$; Ferring, Saint-Prex, Switzerland) was used. All fertilized embryos were cultured to the blastocyst stage in sequential G1/ G2 media (Vitrolife, Gothenburg, Sweden). Good quality embryos having appropriate morphology of the trophectoderm and inner cell mass and fewer fragmentations of blastomeres were selected and vitrified for elective FET. Two to 3 months after the oocyte pick up, oral estradiol valerate was administered in gradually increasing quantity for endometrial preparation (progynova 2-6 $\mathrm{mg}$ /day; Bayer Schering Pharma, Berlin, Germany) after pituitary luteal down-regulation with preliminary $\mathrm{GnRH}$ agonist (lucrin, $0.1 \mathrm{mg} /$ day; Abbott, Chicago, IL, USA). At menstrual day 15, endometrial thickness (EMT) was evaluated. In cases with EMT over 7-8 mm, vaginal progesterone gel (Crinone $90 \mathrm{mg} /$ day; Merck Serono) and oral estradiol valerate were administered for inducing the secretory phase of the endometrium. In cases where EMT did not reach $7 \mathrm{~mm}$, oral estradiol valerate was administered continuously and EMT was rechecked later. Vaginal progesterone was administered after EMT was confirmed to be suitable. Blastocysts were warmed and transferred under transabdominal ultrasound guidance using a soft embryo transfer catheter (Cook Medical, Spencer, IN, USA).

\section{Outcome measures and following procedures}

Twelve days after the embryo transfer, serum $\beta$-hCG was checked for confirmation of pregnancy. Progesterone support was continued until 10 gestational weeks in women who conceived. In the non-pregnant group, a second embryo transfer or a second COS cycle was conducted (Fig. 1).

\section{Statistical analyses}

All patients were retrospectively divided into the pregnant and the non-pregnant groups after FET. The variables under study were compared between the two groups by Wilcoxon rank sum test for independent samples using PASW version 18.0 (SPSS Inc., Chicago, IL, USA). The data were expressed as mean \pm standard deviation unless specified otherwise. The value of $p<0.05$ was considered statistically significant.

\section{Results}

In this study, we analyzed the difference in various clinical parameters in the freeze-all policy between the pregnant group and the non-pregnant group through a single cycle of COS composed of 3 cycles of FET.

Totally, 153 FETs were carried out in 89 patients (Fig. 1). Patient characteristics and outcomes of COS are presented in Table 1. After 


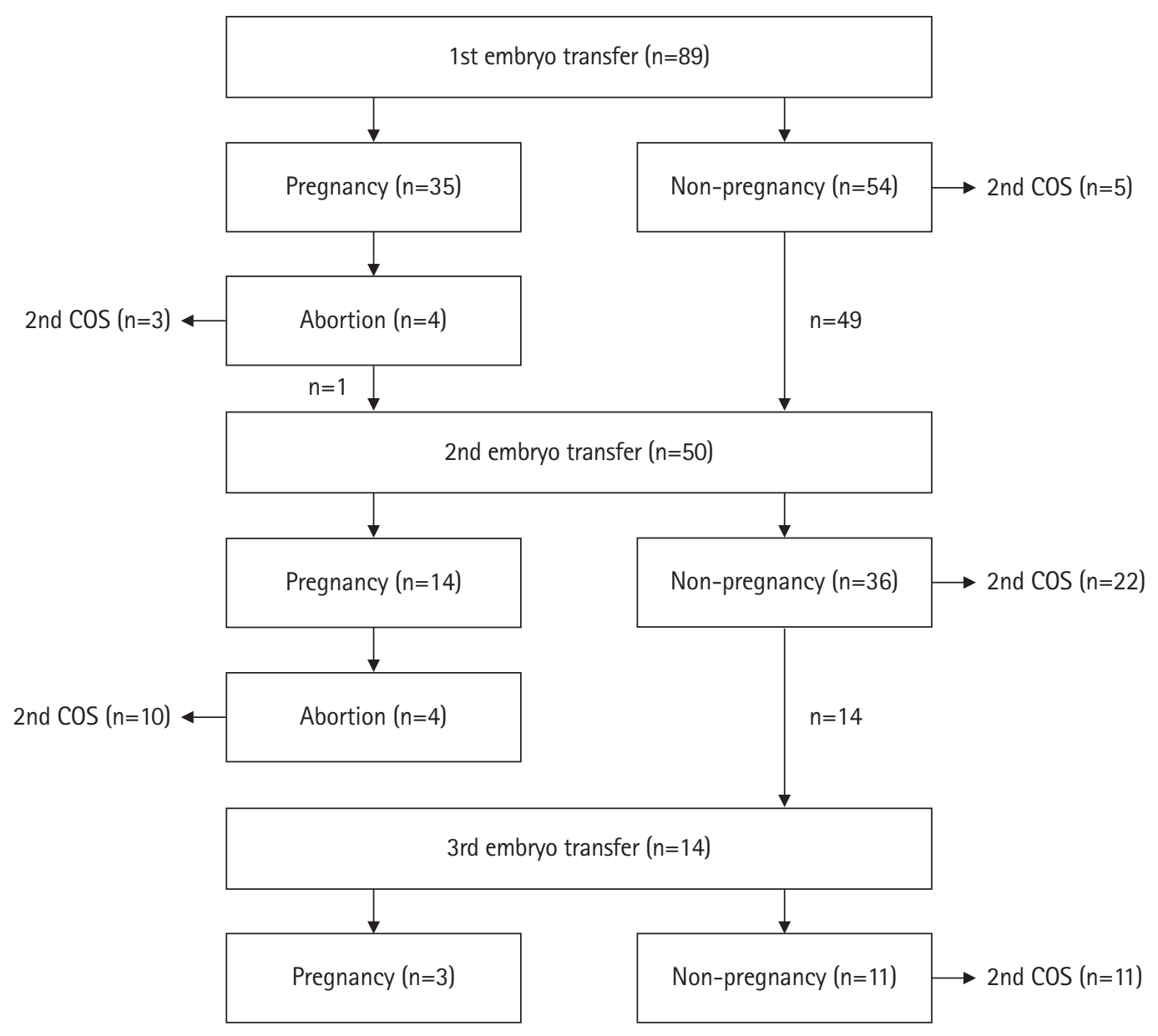

Fig. 1. Flow diagram of the inclusion cycles. In this study, 153 embryo transfer cycles in 89 infertile women were analyzed. The second FET cycle was conducted in 50 patients and the third FET cycle was conducted in 14 patients. In case of no remnant frozen embryos, a second COS was started. FET, frozen-thawed embryo transfer; COS, controlled ovarian stimulation.

the first FET cycle, 35 patients (39.3\%) became pregnant. Table 2 shows the comparison of parameters between the pregnant group and the non-pregnant group after the first FET cycle. Significant differences were observed in the number of matured oocytes, the number of fertilized oocytes, the number of frozen blastocysts, and the number of transferred embryos between the two groups. Among the 35 pregnant patients, 2 patients (5.7\%) had a spontaneous abortion and 2 patients (5.7\%) experienced chemical pregnancy. The second FET cycle was carried out in 50 patients. Fourteen patients $(28.0 \%)$ became pregnant after the second cycle. Among these 14 pregnant patients, 3 patients (21.4\%) experienced chemical pregnancy and 1 patient $(7.1 \%)$ was diagnosed with a missed abortion.

Table 3 shows the comparison of parameters between the pregnant group and the non-pregnant group after all FET cycles. Through a single cycle of COS that consisted of 3 cycle of FET, 52 patients became pregnant, while 37 patients did not become preg- nant. Significant differences were observed in the number of matured oocytes, the number of fertilized oocytes, the number of frozen blastocysts, and the number of transferred embryos. In particular, the number of frozen blastocysts in the pregnant group was almost twice that in the non-pregnant group (5.6 \pm 3.1 vs. $2.8 \pm 1.9, p<0.001$ ).

Fig. 2 shows the area under curve (AUC) of the receiver operating characteristic (ROC) curve for the number of matured oocytes, fertilized oocytes, and frozen blastocysts in the cumulative pregnancy cycle (Fig. 2A) and in the pregnancy cycle after the first FET (Fig. 2B). The AUC of the ROC curve in the cumulative pregnancy group was 0.801 for the 4 frozen blastocysts (95\% confidence interval [CI], 0.625-0.819), 0.713 for the 13 fertilized oocytes ( $95 \% \mathrm{CI}, 0.540-0.747$ ), and 0.710 for the 8 matured oocytes (95\% CI, 0.525-0.733) (Fig. 2A). The AUC of the ROC curve in the pregnancy group after the first FET cycle was 0.730 for the 4 frozen blastocysts (95\% CI, 0.588-0.788), 0.649 for the 
13 fertilized oocytes ( $95 \%$ CI, $0.548-0.753$ ), and 0.634 for the 14 matured oocytes (95\% CI, 0.524-0.733) (Fig. 2B). The AUC of the ROC curve for the 4 frozen blastocysts was 0.801 in the pregnant group.

\section{Discussion}

This study was conducted to investigate the factors that affect the pregnancy rates of the FET cycle in the freeze-all policy. We analyzed the difference in various clinical parameters in the freeze-all policy between the pregnant group and the non-pregnant group through a single cycle of COS composed of 3 cycles of FET. And carried out the freeze-all policy in the normal responders after informed consent was obtained from the patients. Currently, FET is being performed in extra frozen embryos due to the legislation changes such as medical insurance coverage in South Korea.

Introduction of vitrification and development of $\mathrm{GnRH}$ antagonist protocol are the foundations of the freeze-all policy. Vitrification as a method of cryopreservation of embryos improved the survival rate of thawing embryos. By using $\mathrm{GnRH}$ agonist triggering in the $\mathrm{GnRH}$ antagonist protocol, the ovarian hyperstimulation syndrome (OHSS) risk is almost removed from the assisted reproductive technology procedure [3].
Table 1. Patients characteristics and COS outcomes

\begin{tabular}{lc}
\hline Characteristic & Value \\
\hline Patient & $89(100)$ \\
Age $(\mathrm{yr})$ & $33.6 \pm 3.6$ \\
BMI $\left(\mathrm{kg} / \mathrm{m}^{2}\right)$ & $22.9 \pm 3.7$ \\
Primary infertility & $52(58.4)$ \\
Secondary infertility & $37(41.6)$ \\
Duration of infertility (mo) & $47.1 \pm 30.5$ \\
Basic laboratory findings & \\
FSH (mIU/mL) & $6.9 \pm 4.6$ \\
LH (mIU/mL) & $8.1 \pm 13.8$ \\
Prolactin (ng/mL) & $15.9 \pm 9.4$ \\
AMH (ng/mL) & $5.2 \pm 4.1$ \\
COS outcomes & \\
Total dose of FSH (IU) & $3,056.0 \pm 1,102.9$ \\
Total dose of LH (IU) & $1,229.8 \pm 1,178.3$ \\
Oocytes retrieved & $14.1 \pm 6.9$ \\
Oocytes matured & $11.4 \pm 6.1$ \\
Oocytes fertilized & $10.3 \pm 5.6$ \\
Blastocysts frozen & $4.4 \pm 3.0$ \\
\hline
\end{tabular}

Values are presented as number (\%) or mean \pm standard deviation. $\mathrm{COS}$, controlled ovarian stimulation; $\mathrm{BMI}$, body mass index; $\mathrm{FSH}$, folliclestimulating hormone; $\mathrm{LH}$, luteinizing hormone; $\mathrm{AMH}$, anti-Müllerian hormone.

Table 2. Clinical and COS parameters according to pregnancy or not after first FET

\begin{tabular}{|c|c|c|c|}
\hline Clinical and COS parameter & Pregnant group $(n=35)$ & Non-pregnant group $(n=54)$ & $p$-value \\
\hline Primary infertility & $18(51.4)$ & $34(63.0)$ & $0.105^{\mathrm{a})}$ \\
\hline Secondary infertility & $17(48.6)$ & $20(37.0)$ & \\
\hline Age $(y r)$ & $33.5 \pm 3.4$ & $33.7 \pm 3.8$ & 0.987 \\
\hline BMI $\left(\mathrm{kg} / \mathrm{m}^{2}\right)$ & $22.4 \pm 3.2$ & $23.1 \pm 3.9$ & 0.457 \\
\hline Duration of infertility (mo) & $40.5 \pm 28.6$ & $51.3 \pm 31.2$ & 0.103 \\
\hline \multicolumn{4}{|l|}{ Basic laboratory findings } \\
\hline $\mathrm{FSH}(\mathrm{mlU} / \mathrm{mL})$ & $7.0 \pm 4.5$ & $6.8 \pm 4.7$ & 0.564 \\
\hline $\mathrm{LH}(\mathrm{mlU} / \mathrm{mL})$ & $8.5 \pm 15.7$ & $7.9 \pm 12.6$ & 0.253 \\
\hline Prolactin (ng/mL) & $16.6 \pm 11.6$ & $15.5 \pm 7.7$ & 0.920 \\
\hline AMH (ng/mL) & $4.7 \pm 3.5$ & $5.65 \pm 4.6$ & 0.591 \\
\hline \multicolumn{4}{|l|}{ COS outcomes } \\
\hline Total dose of FSH (IU) & $3,034.3 \pm 1,119.8$ & $3,070.0 \pm 1,102.2$ & 0.468 \\
\hline Total dose of LH (IU) & $1,125.0 \pm 865.5$ & $1,295.6 \pm 1,342.0$ & 0.492 \\
\hline Oocytes retrieved & $15.6 \pm 7.1$ & $13.1 \pm 6.7$ & 0.107 \\
\hline Oocytes matured & $13.1 \pm 6.6$ & $10.3 \pm 5.5$ & 0.037 \\
\hline Oocytes fertilized & $12.1 \pm 6.4$ & $9.1 \pm 4.8$ & 0.020 \\
\hline Blastocysts frozen & $6.1 \pm 3.5$ & $3.4 \pm 2.0$ & $<0.001$ \\
\hline Blastocysts transferred & $2.1 \pm 0.1$ & $1.7 \pm 0.1$ & 0.009 \\
\hline
\end{tabular}

Values are presented as number (\%) or mean \pm standard deviation.

COS, controlled ovarian stimulation; FET, frozen embryo transfer; BMI, body mass index; FSH, follicle-stimulating hormone; LH, luteinizing hormone; $\mathrm{AMH}$, anti-Müllerian hormone.

Wilcoxon rank sum test. ${ }^{\text {a) }}$ Fisher exact test. 
Table 3. Clinical and COS parameters according to pregnancy or not after all FET

\begin{tabular}{|c|c|c|c|}
\hline Clinical and COS parameter & Pregnant group $(n=52)$ & Non-pregnant group $(n=37)$ & $p$-value \\
\hline Primary infertility & $30(57.7)$ & $22(59.5)$ & $0.784^{a}$ \\
\hline Secondary infertility & $22(42.3)$ & $15(40.5)$ & \\
\hline Age (yr) & $33.3 \pm 3.4$ & $34.0 \pm 3.9$ & 0.677 \\
\hline $\mathrm{BMI}\left(\mathrm{kg} / \mathrm{m}^{2}\right)$ & $22.9 \pm 4.0$ & $22.8 \pm 3.1$ & 0.836 \\
\hline Duration of infertility (mo) & $44.3 \pm 29.6$ & $51.1 \pm 31.7$ & 0.339 \\
\hline \multicolumn{4}{|l|}{ Basic laboratory findings } \\
\hline $\mathrm{FSH}(\mathrm{mlU} / \mathrm{mL})$ & $7.1 \pm 4.4$ & $6.6 \pm 5.0$ & 0.307 \\
\hline $\mathrm{LH}(\mathrm{mlU} / \mathrm{mL})$ & $8.1 \pm 14.3$ & $8.1 \pm 13.3$ & 0.245 \\
\hline Prolactin $(\mathrm{ng} / \mathrm{mL})$ & $16.3 \pm 10.1$ & $15.3 \pm 8.3$ & 0.666 \\
\hline AMH (ng/mL) & $5.4 \pm 4.1$ & $4.9 \pm 4.3$ & 0.439 \\
\hline \multicolumn{4}{|l|}{ COS outcomes } \\
\hline Total dose of FSH (IU) & $3,008.2 \pm 948.6$ & $3,123.1 \pm 1,300.4$ & 0.767 \\
\hline Total dose of LH (IU) & $1,109.4 \pm 946.0$ & $1,995.0 \pm 1,436.1$ & 0.857 \\
\hline Oocytes retrieved & $15.4 \pm 6.8$ & $12.3 \pm 6.7$ & 0.031 \\
\hline Oocytes matured & $12.9 \pm 6.1$ & $9.2 \pm 5.4$ & 0.006 \\
\hline Oocytes fertilized & $11.8 \pm 5.8$ & $8.2 \pm 4.7$ & 0.004 \\
\hline Blastocysts frozen & $5.6 \pm 3.1$ & $2.8 \pm 1.9$ & $<0.001$ \\
\hline Bblastocysts transferred & $2.0 \pm 0.5$ & $1.7 \pm 0.5$ & 0.001 \\
\hline
\end{tabular}

Values are presented as number (\%) or mean \pm standard deviation.

COS, controlled ovarian stimulation; FET, frozen embryo transfer; BMI, body mass index; FSH, follicle-stimulating hormone; LH, luteinizing hormone; $\mathrm{AMH}$, anti-Müllerian hormone.

Wilcoxon rank sum test. ${ }^{\text {a) }}$ Fisher exact test.
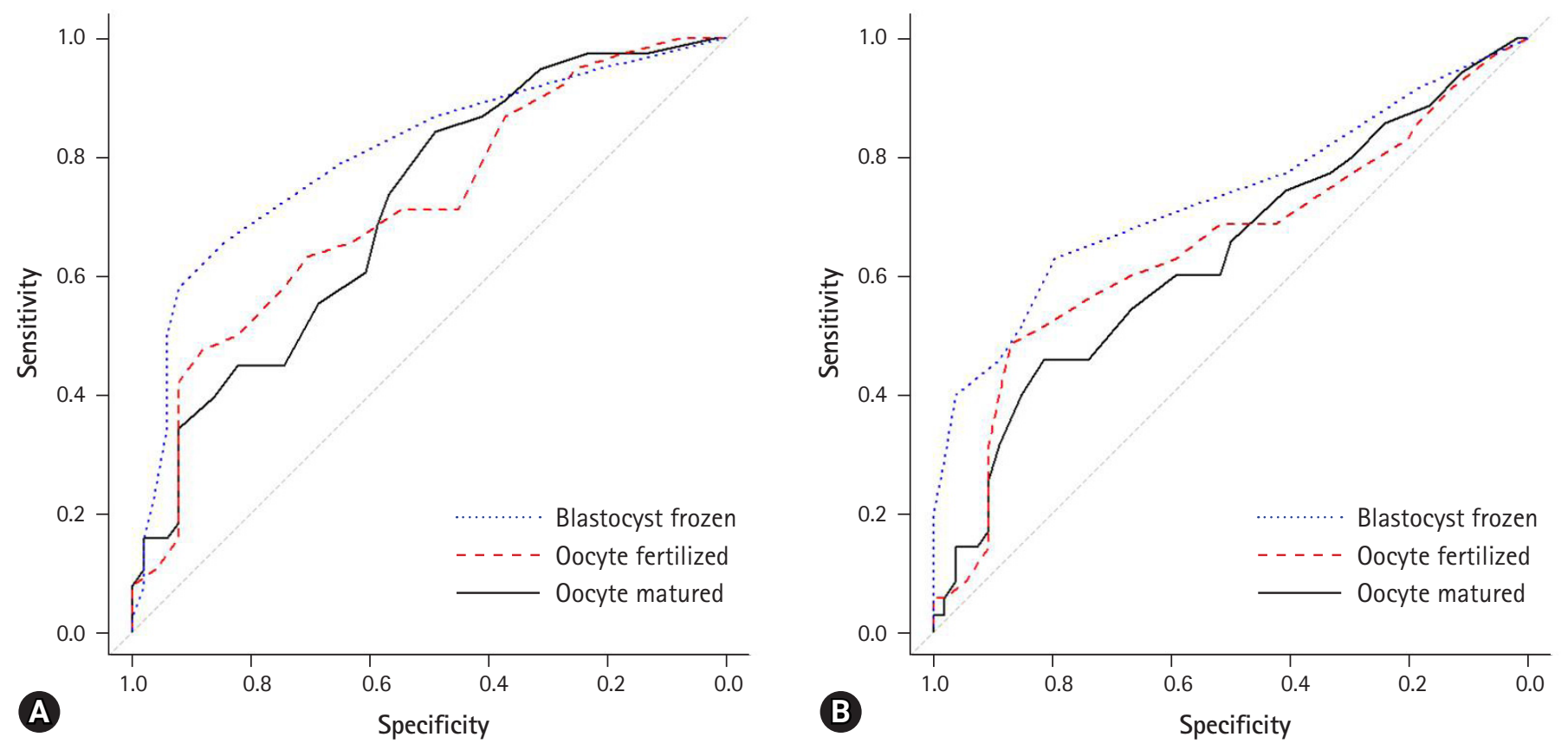

Fig. 2. ROC curve of the cumulative pregnancy group (A) and the pregnancy group after the first frozen embryo transfer (B). (A) AUC of the ROC curve of the cumulative pregnancy group: 0.801 for the 4 frozen blastocysts $(95 \% \mathrm{Cl}, 0.625-0.819), 0.713$ for the 13 fertilized oocytes ( $95 \% \mathrm{Cl}, 0.540-0.747)$, and 0.710 for the 8 matured oocytes $(95 \% \mathrm{Cl}, 0.525-0.733)$. (B) AUC of the ROC curve of the pregnancy group for the first frozen embryo transfer: 0.730 for the 4 frozen blastocysts $(95 \% \mathrm{Cl}, 0.588-0.788), 0.649$ for the 13 fertilized oocytes $(95 \% \mathrm{Cl}, 0.548-0.753)$, and 0.634 for the 14 matured oocytes $(95 \% \mathrm{Cl}, 0.524-0.733)$. ROC, receiver operating characteristic; AUC, area under curve; $\mathrm{Cl}$, confidence interval. 
Apart from the decreased risk of OHSS, the freeze-all policy has several strengths. A meta-analysis comparing the fresh transfers and the FET cycles showed significantly higher implantation rates and clinical and ongoing pregnancy rates in the FET cycles [7]. The suggested mechanisms for the poor clinical outcomes in the fresh cycles are abnormal hormonal milieu and the suboptimal endometrial development in the COS cycles [4]. Moreover, the rate of ectopic pregnancy is reduced $[8,9]$ and better perinatal outcomes have been reported in FET cycles [10,11].

However, there are several critical opinions about the freeze-all policy. A meta-analysis about the results of the freeze-all policy included only three small studies and the total number of patients included in the analysis was only 633 [12]. The studies included in this meta-analysis were highly heterogeneous in terms of the types of patients and some of the technical aspects [12]. To confirm the advantages of the freeze-all policy, further large-scale research with homogeneous criteria is needed.

There are some obstacles to the adaptation of the freeze-all policy to the real clinical practice. The policy needs changes in the current IVF practice and optimization of the cryopreservation techniques [4]. In an institute having suboptimal culture techniques, the possibility of cancellation of embryo transfer after COS would be increased. To apply the freeze-all policy to general infertility clinics, it has to be preceded by quality control of the culture technique. After quality control, the strengths of the freeze-all policy, such as better pregnancy rates and lower ectopic pregnancy rates, could be achieved, particularly in normal responders. Moreover, the freeze-all policy is a cost-effective strategy when compared to the fresh embryo transfer [13]. Thus, if adequate number of oocytes or embryos were retrieved, the freezeall policy is a better option than the fresh embryo transfer in normal responders.

In this study, the AUC of the ROC curve was 0.801 for cumulative pregnancy group when the cut-off value of the number of frozen blastocysts was 4 . To the best of our knowledge, there has been no report suggesting the cut-off value for the freeze-all policy as a predictive parameter for pregnancy.

The outcomes of IVF could be different in low or poor responders. Roque et al. [14] reported that the freeze-all policy may be related to better IVF outcomes in normal responders, but these potential advantages decrease with worsening ovarian response. In patients with 4-9 oocytes retrieved, the implantation rate and the ongoing pregnancy rate showed no difference between the fresh embryo transfer and the FET group. Meanwhile, patients with 10-15 retrieved oocytes showed significant differences in the implantation rate and the ongoing pregnancy rate between the fresh transfer group and the FET group. The FET group showed better outcomes (implantation rate of $30.1 \%$ in the FET group and $22.1 \%$ in the fresh embryo transfer, $p=0.028$; ongoing pregnancy rate of $47 \%$ in the FET group and $34 \%$ in the fresh embryo transfer, $p=0.021)$. Moreover, in poor responders, cycle cancellation rates could be increased.

On the contrary, the strengths of the freeze-all policy such as better pregnancy rates and perinatal outcomes and lesser ectopic pregnancy rates might also be similar for the poor responders. In case of the poor responders who need uterine surgery for improving the possibility of implantation, the freeze-all policy could avoid unnecessary operation. Usually, in older age groups, increased incidence of benign uterine disease is observed and the chances of poor response to COS are increased. If an adequate number of embryos could be obtained, gynecologic operation could be conducted after freezing all the embryos. In case of failure to obtain adequate embryos, benign uterine disease could be managed by other treatment modalities. The freeze-all policy is also suitable for preimplantation genetic testing, which is applied more commonly in the older age group. Hence, the freeze-all policy could have several advantages in normal as well as poor responders.

This study suggested a cut-off value of the number of oocytes and blastocysts for the pregnant group in the freeze-all policy. However, there are critical limitations to this study. The number of patients included in this study was relatively small, and the study was carried out retrospectively. The possible causes of repeated implantation failure, such as intrauterine adhesion and thin endometrium were considered in the exclusion criteria. However, the factors associated with immunologic or thrombotic tendencies were not included in the screening. In addition, a dramatic decrease was observed in the pregnancy rates in the second cycle of FET. This finding may be because the embryos with poorer quality than those from the first cycle had been transferred. Only the good quality embryos were frozen according to the morphological criteria. However, the quality of the embryos in the same grade of the morphological criteria may vary. All embryos were cultured to the blastocyst stage at our center. Hence, information about the prediction of pregnancy in day 3 embryo transfer could not be obtained.

Despite these limitations, this study might provide significant information about the predictive value of clinical parameters and results of COS for pregnancy rates in the freeze-all policy.

In this study, we confirmed that the number of matured oocytes, fertilized oocytes, and frozen blastocysts might be predictive factors for pregnancy in the freeze-all policy. 


\section{Acknowledgments}

\section{Conflicts of interest}

No potential conflict of interest relevant to this article was reported.

\section{Funding}

This work was supported by clinical research grant from Pusan National University Hospital in 2019.

\section{ORCID}

Seo Yoon Hwang, https://orcid.org/0000-0001-5495-7236

Eun Hye Jeon, https://orcid.org/0000-0003-4185-2915

Seung Chul Kim, https://orcid.org/0000-0002-8174-9931

Jong Kil Joo, https://orcid.org/0000-0002-6338-1512

\section{References}

1. Shapiro BS, Daneshmand ST, Garner FC, Aguirre M, Hudson C. Clinical rationale for cryopreservation of entire embryo cohorts in lieu of fresh transfer. Fertil Steril 2014;102:3-9.

2. Maheshwari A, Pandey S, Shetty A, Hamilton M, Bhattacharya S. Obstetric and perinatal outcomes in singleton pregnancies resulting from the transfer of frozen thawed versus fresh embryos generated through in vitro fertilization treatment: a systematic review and meta-analysis. Fertil Steril 2012;98:368-77.

3. Devroey P, Polyzos NP, Blockeel C. An OHSS-Free Clinic by segmentation of IVF treatment. Hum Reprod 2011;26:2593-7.

4. Blockeel C, Drakopoulos P, Santos-Ribeiro S, Polyzos NP, Tournaye $\mathrm{H}$. A fresh look at the freeze-all protocol: a SWOT analysis. Hum Reprod 2016;31:491-7.

5. Veleva Z, Orava M, Nuojua-Huttunen S, Tapanainen JS, Martikainen $\mathrm{H}$. Factors affecting the outcome of frozen-thawed em- bryo transfer. Hum Reprod 2013;28:2425-31.

6. Son JB, Jeong JE, Joo JK, Na YJ, Kim CW, Lee KS. Measurement of endometrial and uterine vascularity by transvaginal ultrasonography in predicting pregnancy outcome during frozen-thawed embryo transfer cycles. J Obstet Gynaecol Res 2014;40:1661-7.

7. Roque M, Lattes K, Serra S, Solà I, Geber S, Carreras R, et al. Fresh embryo transfer versus frozen embryo transfer in in vitro fertilization cycles: a systematic review and meta-analysis. Fertil Steril 2013;99:156-62.

8. Ishihara O, Kuwahara A, Saitoh H. Frozen-thawed blastocyst transfer reduces ectopic pregnancy risk: an analysis of single embryo transfer cycles in Japan. Fertil Steril 2011;95:1966-9.

9. Shapiro BS, Daneshmand ST, De Leon L, Garner FC, Aguirre M, Hudson C. Frozen-thawed embryo transfer is associated with a significantly reduced incidence of ectopic pregnancy. Fertil Steril 2012;98:1490-4.

10. Ishihara O, Araki R, Kuwahara A, Itakura A, Saito H, Adamson GD. Impact of frozen-thawed single-blastocyst transfer on maternal and neonatal outcome: an analysis of 277,042 single-embryo transfer cycles from 2008 to 2010 in Japan. Fertil Steril 2014;101:128-33.

11. Kalra SK, Ratcliffe SJ, Coutifaris C, Molinaro T, Barnhart KT. Ovarian stimulation and low birth weight in newborns conceived through in vitro fertilization. Obstet Gynecol 2011; 118:863-71.

12. Basile N, Garcia-Velasco JA. The state of "freeze-for-all" in human ARTs.J Assist Reprod Genet 2016;33:1543-50.

13. Roque M, Valle M, Guimarães F, Sampaio M, Geber S. Cost-effectiveness of the freeze-all policy. JBRA Assist Reprod 2015;19:125-30.

14. Roque M, Valle M, Guimarães F, Sampaio M, Geber S. Freezeall cycle for all normal responders? J Assist Reprod Genet 2017;34:179-85. 